\title{
Quantitative In Situ SEM High Cycle Fatigue: the Critical Role of Oxygen on Nanoscale-Void- Controlled Nucleation and Propagation of Small Cracks in Ni Microbeams
}

\author{
Alejandro Barrios ${ }^{1}$, Saurabh Gupta ${ }^{1}$, Gustavo M. Castelluccio ${ }^{2}$, and Olivier N. Pierron ${ }^{1, *}$ \\ ${ }^{1}$ G.W. Woodruff School of Mechanical Engineering, Georgia Institute of Technology, Atlanta, \\ GA, 30332-0405, USA \\ ${ }^{2}$ Cranfield University, Bedfordshire, MK43 0AL, UK
}

KEYWORDS: nanomechanics, quantitative in situ SEM, high cycle fatigue, small cracks, voids, environmental effects 
ABSTRACT: This letter presents a quantitative in situ scanning electron microscope (SEM) nanoscale high and very high cycle fatigue $(\mathrm{HCF} / \mathrm{VHCF})$ investigation of Ni microbeams under bending, using a MEMS microresonator as an integrated testing machine. The novel technique highlights ultraslow fatigue crack growth (average values down to $\sim 10^{-14} \mathrm{~m} / \mathrm{cycle}$ ) that has heretofore not been reported and that indicates a discontinuous process; it also reveals strong environmental effects on fatigue lives that are three orders of magnitude longer in vacuum than in air. This ultraslow fatigue regime does not follow the well documented fatigue mechanisms that rely on the common crack tip stress intensification, mediated by dislocation emission and associated with much larger crack growth rates. Instead, our study reveals fatigue nucleation and propagation mechanisms that mainly result from room temperature void formation based on vacancy condensation processes that are strongly affected by oxygen. This study therefore shows significant size effects governing the bending high / very high cycle fatigue behavior of metals at the micro / nano scales, whereby the stress concentration effect at the tip of a growing small fatigue crack is assumed to be greatly reduced by the effect of the bending-induced extreme stress gradients, which prevents any significant cyclic crack tip opening displacement. In this scenario, ultraslow processes relying on vacancy formation at the subsurface or in the vicinity of a crack tip and subsequent condensation into voids become the dominant fatigue mechanisms. 
Understanding and controlling the fatigue properties of micro- and nano-scale structural materials has become an essential pursuit, as these micro/nano materials become more prominent in our daily lives. The Internet of Things is expected to connect 24 billion devices by $2020,{ }^{1}$ many of which will include actuators and sensors fabricated with microelectromechanical systems (MEMS) technology, thereby including moving micro/nano components subjected to large numbers of cycles throughout their targeted lifetimes. The size effects associated with the fatigue properties of these components must therefore be investigated. Silicon is still the main structural material employed in commercial MEMS devices, despite its inherent brittleness (fracture toughness around $1 \mathrm{MPa} \cdot \mathrm{m}^{1 / 2}$ ). Other structural materials, including metals, are likely to be used in the near future, especially for harsh applications where brittle materials may not be suitable. ${ }^{2}$ For example, metallic MEMS films with excellent thermal and mechanical stability were recently synthesized, and they are promising candidates for the next generation of metal MEMS devices. ${ }^{3}$ Therefore, new techniques are required to characterize fatigue damage and its size effects at the nanoscale in metallic micro-components under loading conditions relevant to MEMS devices. ${ }^{4-15}$ This study introduces a novel quantitative in situ scanning electron microscope (SEM) nanoscale high / very high cycle bending fatigue characterization study of electroplated Ni microbeams, using a MEMS microresonator as an integrated testing machine. ${ }^{16}$ The technique allows accurate measurement of ultraslow fatigue crack growth (average values down to $10^{-14} \mathrm{~m} / \mathrm{cycle}$ ) that have heretofore not been reported, and highlights strong environmental effects on fatigue lives that are three orders of magnitude longer in vacuum than in air. This ultraslow fatigue regime does not follow the well documented fatigue mechanisms that rely on the common crack tip stress intensification associated with crack growth rates mediated by dislocation emission $\left(>\sim 10^{-10}\right.$ $\mathrm{m} /$ cycle). ${ }^{17}$ Instead, our study reveals fatigue nucleation and propagation mechanisms that mainly 
rely on room temperature vacancy condensation leading to voids whose nucleation process is strongly affected by oxygen.

The MEMS microresonator shown in Figure 1(a) consists of a Ni microbeam clamped to the substrate on one side and connected to a plate-shaped mass with two sets of interdigitated fingers (comb structures). ${ }^{4}$ These devices were fabricated with the MetalMUMPs process. ${ }^{18}$ The

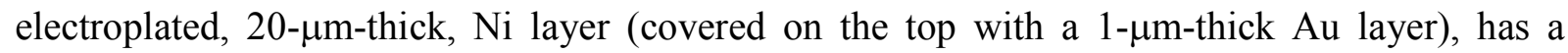
columnar microstructure, with grain diameter of approximately 1-2 $\mu \mathrm{m} .{ }^{4}$ Its mechanical tensile properties were previously measured using micro-tensile testing: ${ }^{4}$ the $0.2 \%$ yield stress is $\sim 650$ $\mathrm{MPa}$, and the tensile strength is $\sim 875 \mathrm{MPa}$. This fatigue test micro-machine allows cyclic loading of the microbeam under fully-reversed, in-plane bending with low plastic strain amplitudes, $\varepsilon_{\mathrm{pa}}$ $\left(\varepsilon_{\mathrm{pa}}<10^{-3}\right),{ }^{16}$ at a frequency fixed by the microresonator's resonance frequency, $f_{0}\left(f_{0} \sim 8 \mathrm{kHz}\right){ }^{16}$, ${ }^{19,20}$ It can therefore characterize the high and very high cycle fatigue regimes (HCF/VHCF) of small-scale materials under relevant loading conditions for MEMS applications, requiring as little as $3.5 \mathrm{~h}$ to accumulate $10^{8}$ cycles. The fatigue damage developing on both sidewalls of the microbeam (see example in Figures 1(b),(e), and (f)) results in a decrease in stiffness and therefore $f_{0}$, which is measured throughout the fatigue test and used as a metric to define fatigue damage. ${ }^{16 \text {, }}$ ${ }^{19,20}$ For the microbeams used in this study, the fatigue life, $N_{\mathrm{f}}$, is defined as the number of cycles to reach a $10 \%$ decrease in $f_{0}$, which has been shown to correlate to a $2-\mu \mathrm{m}$-long crack on each side. ${ }^{16}$ Unlike bulk ultrasonic fatigue testing, the small specimen size and large surface-to-volume ratio prevents any significant heating of the microbeam at resonance, which would be captured by a large measured reversible change in $f_{0}\left(\right.$ a $50^{\circ} \mathrm{C}$ increase in temperature leads to a decrease in $f_{0}$ of $\sim 1 \%) .{ }^{4,20}$ This fatigue technique is therefore perfectly suited to investigate size effects relevant 
to the long term reliability of small-scale metallic components, such as the nucleation and growth of microstructurally small fatigue cracks (as the fatigue cracks are commensurate with the microbeam's grain size) under extreme stress gradients imposed by the width of the microbeam (the normalized stress gradient of the microbeam used in this study is: $\eta=\frac{1}{\sigma_{\max }} \frac{d \sigma}{d x}=17 \% / \mu m$ ). ${ }^{19}$ For the first time, this technique has been successfully employed to perform fatigue tests inside a SEM (see section A in the Supporting Information), thereby providing unprecedented levels of details regarding the fatigue damage of microbeams, and a robust quantification of the role of air on the nucleation and growth of these small cracks. For example, the in situ SEM fatigue tests allow for a much better observation of the evolution of fatigue damage (fatigue crack formation and evolution of the crack shape along the sidewalls) throughout the fatigue test, whereas our previous fatigue tests would mainly allow post mortem SEM examination.

Figure 1(c) shows the stress-life $\left(\sigma_{\mathrm{a}}-N_{\mathrm{f}}\right)$ fatigue curves for the Ni microbeams tested in vacuum (in situ SEM experiments) and in air (ex situ, at $30^{\circ} \mathrm{C}, 50 \% \mathrm{RH}$ and $80^{\circ} \mathrm{C}, 90 \% \mathrm{RH}$ ). ${ }^{16}$ The stress amplitude $\sigma_{\mathrm{a}}$ refers to the initial stress amplitude value at the edge of the microbeam. The empty symbols denote runouts, while the solid ones represent fatigue failure (defined as a $10 \%$ decrease in resonance frequency, $f_{0}$ ). The curves highlight three orders of magnitude longer fatigue lives in vacuum for $\sigma_{\mathrm{a}}=450 \mathrm{MPa}\left(N_{\mathrm{f}} \sim 10^{8}\right.$ cycles in vacuum vs $\sim 10^{5}$ cycles in air). Below that stress level, specimens tested in vacuum do not fail in the very high cycle fatigue regime, while the ones tested in air can sustain more than $10^{8}$ cycles for $\sigma_{\mathrm{a}}=300 \mathrm{MPa}$. No fatigue failure was observed below that stress level (runouts $>3 \times 10^{9}$ cycles in air). Note that for macroscopic specimens, failure usually refers to the number of cycles required to propagate a fatigue crack until full separation. 
Instead, here $N_{\mathrm{f}}$ refers to the number of cycles to nucleate a crack and grow it to a size of $2 \mu \mathrm{m}$, while it is still microstructurally small. Our previous ex situ studies showed that the extreme stress gradients (imposed by the microbeam's width) strongly influence the crack growth rates of these microstructurally small cracks and therefore $N_{\mathrm{f} .}{ }^{16,19,20}$ In this study, our in situ SEM fatigue experiments unambiguously demonstrate that the longer $N_{\mathrm{f}}$ measured in vacuum is both due to the significant role of air in both fatigue crack nucleation and propagation, as highlighted next.

Figure 2(a) shows the evolution of $f_{0}$ during an in situ SEM fatigue test performed at $\sigma_{\mathrm{a}}=470$ $\operatorname{MPa}\left(N_{\mathrm{f}}=5.6 \times 10^{7}\right)$, compared to that of an ex situ test performed in air at $\sigma_{\mathrm{a}}=400 \mathrm{MPa}\left(N_{\mathrm{f}}=3.7\right.$ $\times 10^{6}$ ). A series of SEM images taken throughout the vacuum fatigue test (Figure 2(b)-(i)) capture the evolution of fatigue damage along the sidewalls, which correlates to the corresponding decrease in $f_{0}$. The $f_{0}$ evolution curve in vacuum shows a very slow decrease over the first $\sim 3 \times 10^{7}$ cycles that is not observed in air, which instead presents a significant decrease in $f_{0}$ from the onset of the test despite a lower $\sigma_{\mathrm{a}}$. Fatigue extrusions, with an approximated maximum height of $1 \mu \mathrm{m}$ (based on top-down SEM images not shown here) are observed after $8.2 \times 10^{5}$ cycles (see Figure 2(c)). Between $8.2 \times 10^{5}$ and $3.2 \times 10^{7}$ cycles, the number of extrusions along the sidewall increases significantly (see Figures 2(c)-(g)). Some intrusions (or fatigue crack embryos) develop as well at the edges of extrusions (see arrows in Figures 2(c)-(g), see also arrows in Figure 1(b)), resulting in a slow decrease in $f_{0}$ (see Figure 2(a)), but only after $4.3 \times 10^{7}$ cycles can a clear fatigue crack be observed (see arrow in Figure 2(h)). Hence the nucleation of a fatigue crack occurred between 3.2 and $4.3 \times 10^{7}$ cycles, corresponding to a decrease in $f_{0}$ between 2 and $4 \%$. Our quasicontinuous observation of the fatigue damage along the sidewall also provides information 
regarding the evolution of the crack shape, from a 3D, "penny-shaped" crack to a longer crack that, in most cases, spans the entire thickness of the microbeam (2D crack; see for example Fig. $1(\mathrm{e})$ and (f)). In contrast, a $4 \%$ decrease in $f_{0}$ occurs after only $2.3 \times 10^{6}$ cycles for the fatigue test in air shown in Figure 2(a), which strongly suggests that the air accelerates the fatigue crack nucleation process by at least one order of magnitude.

The test shown in Figure 3 confirms these findings, and consists of cycling a microbeam first in vacuum at $\sigma_{\mathrm{a}}=420 \mathrm{MPa}$ for $6.4 \times 10^{7}$ cycles, followed by cycling in air at $\sigma_{\mathrm{a}}=360 \mathrm{MPa}$ for $\sim 1.5 \times 10^{7}$ cycles, and further cycling again in vacuum (at $\left.\sigma_{\mathrm{a}}=420 \mathrm{MPa}\right)$. SEM images at the end of each segment were taken to observe crack initiation and growth. Figure 3(a) shows that $f_{0}$ does not decrease in vacuum for the first $6 \times 10^{7}$ cycles, at which point the SEM image (see Figure 3(c)) shows that extrusions were formed, but no fatigue cracks, which is consistent with a constant $f_{0}$. During the fatigue test in air, and despite a lower $\sigma_{\mathrm{a}}, f_{0}$ decreases $4 \%$, which correlates with the initiation and growth of a fatigue crack (see arrow in Figure 3(d)). This crack initiated next to an extrusion formed while cycling in vacuum (see Figure 3(c)). Upon further testing in vacuum, the crack grew further, as evidenced by the decrease in $f_{0}$ shown in Figure 3(a) and the arrows in Figure 3(e). Overall, these results indicate that air plays a crucial role in the formation of fatigue cracks. As shown in Figure 1(c), the larger endurance limit, $\sigma_{e}$, in vacuum $\left(\sigma_{e}\right.$, vacuum $=425 \mathrm{MPa}>\sigma_{\mathrm{e}}$, air $=$ $300 \mathrm{MPa}$ ) is related to the larger fatigue crack initiation threshold in vacuum due to these environmental effects. 
The effect of air on fatigue crack propagation rates for these microbeams was quantified through the following series of experiments. Figure 4(a) shows the $f_{0}$ evolution plot of a fatigue test consisting of cycling a specimen at $\sigma_{\mathrm{a}}=400 \mathrm{MPa}$ in air for $9.3 \times 10^{6}$ cycles, followed by cycling in vacuum until $1.3 \times 10^{8}$ cycles, first at $\sigma_{\mathrm{a}}=350 \mathrm{MPa}$, then at $\sigma_{\mathrm{a}}=385 \mathrm{MPa}$. Two 2D cracks (i.e., SEM examination of the sidewalls confirmed the cracks spanned through the microbeam's thickness), one on each side of the microbeam, developed in air during the first portion of the test (see Figure 4(b)). Based on the measured total crack length at the surface, $2 a$, where $a$ is the crack length, the average crack growth rate is $3 \times 10^{-13} \mathrm{~m} /$ cycle. This rate is extremely low and indicate strong size effects associated with the fatigue behavior of these microbeams. The crack did not extend in vacuum at $\sigma_{\mathrm{a}}=350 \mathrm{MPa}$, but at $\sigma_{\mathrm{a}}=385 \mathrm{MPa}$, the crack grew as shown in Figures 4(d) and (e) (see also Animation 1 in the Supporting Information). The average crack growth rate is $5.5 \times 10^{-15} \mathrm{~m} /$ cycle, which is about 50 times slower than in air. Similar results were obtained in another experiment described in section B of the Supporting Information (see also Animation 2 in the Supporting Information). In air, the average crack growth rate is 2.0 $\times 10^{-12} \mathrm{~m} /$ cycle at $\sigma_{\mathrm{a}}=390 \mathrm{MPa}$, while in vacuum, the rate is 50 times lower $\left(3.7 \times 10^{-14} \mathrm{~m} / \mathrm{cycle}\right)$ at $\sigma_{\mathrm{a}}=360 \mathrm{MPa}$.

Figure 1(d) summarizes all the measured crack growth rates in air and vacuum, based on actual crack length, $a$, measurements (ranging between 1 and $3 \mu \mathrm{m}$ ) with the SEM images (providing a resolution of $\sim 10 \mathrm{~nm}$ ), as a function of $\sigma_{\mathrm{a}}$, confirming the ultralow rates and highlighting the roughly two orders of magnitude larger rates in air compared to vacuum. See also Figure S4 in section $\mathrm{C}$ of the supporting information for a plot of the rates as a function of the crack size. These ultralow crack growth rates were only measured for fatigue cracks that spanned the microbeam's 
thickness (2D cracks), thanks to our in situ SEM technique that allowed meticulous observation of the crack shape evolution. While this crack growth rate measurement technique does not follow the traditional fatigue dimensional requirements, it is based on direct measurement of the crack size, and therefore provides an accurate measurement of the local crack growth rates (at the surface), which are not expected to be much different from the overall crack growth rates of the 2D cracks given the fairly uniform crack fronts observed post mortem. Importantly enough, these rates are of the same order of magnitude as the rates previously calculated based on finite element models linking the decrease in $f_{0}$ to an increase in crack size. ${ }^{16,19}$ However, these new rates are more accurate since they are based on direct measurement of crack size (for 2D cracks), whereas the previously calculated rates relied on simplifying assumptions (such as the assumption of a single crack on each size of the microbeam contributing to the measured decrease in $f_{0}$ ). Overall, these rates are several orders of magnitude lower than that typically measured on macroscopic specimens. Nonetheless, they can be measured thanks to the large testing frequency (in one minute of testing, a rate of $10^{-14} \mathrm{~m} /$ cycle would lead to $\sim 5 \mathrm{~nm}$ crack extension), and to the microscopic size of the specimens. Being significantly less than one interatomic spacing $\left(\sim 2.5 \times 10^{-10} \mathrm{~m}\right)$ per cycle, these rates suggest that crack growth is not a continuous process but instead occurs in between incubation periods. For example, at an average rate of $10^{-14} \mathrm{~m} / \mathrm{cycle}$, it would take 25,000 cycles ( $3 \mathrm{~s}$ of testing) to grow the crack by one interatomic spacing. The fractography results shown next highlight a new fatigue mechanism accounting for this ultraslow fatigue crack growth.

To further understand the mechanisms responsible for the ultra-low crack growth rates, a series of FIB cross-sections (see Figure 5(a) for the orientations of the cuts) were performed on five specimens: three fatigued in air, and two in vacuum. Figures S6 and S7 in section D of the 
Supporting Information, with the low magnification SEM images of the series of parallel cuts, show that the main cracks at the end of these fatigue tests are fairly uniform through the thickness of the microbeam. More importantly, for both testing environments, these FIB cross-sections reveal a tortuous crack path consisting of voids (ranging in size from $\sim 10$ to $\sim 500 \mathrm{~nm}$ ) that are linked together by straight crack paths. Voids are either observed in the vicinity of the cracks (within $\sim 1 \mu \mathrm{m}$; see for example Figures 5(b), (c), (i)), or in the subsurface underneath the extrusions (also within $\sim 1 \mu \mathrm{m}$ of the surface; see Figures $5(\mathrm{k})-(\mathrm{m})$ ). The voids do not appear to form preferentially at grain boundaries (only in Figure 5(i) is a void observed at a grain boundary), and the crack path is mainly transgranular. Figure 5(d) clearly shows two cuboid voids, each $\sim 10$ $\mathrm{nm}$ in size, formed $\sim 500 \mathrm{~nm}$ ahead of the main crack tip, at a $45^{\circ}$ angle with respect of the main crack. A very thin crack appears to be linking the main crack tip to the two voids. Very similar observations can be made at the location of the extrusions, where fatigue crack nucleate. For example, Figure 5(1) shows small voids developing underneath the extrusions, and thin cracks linking them together. For the specimens tested in air, there are many more voids along the crack path (see Figures 5(h) and (i)), as a result of which the crack faces appear to be rougher. See as well Section D in the Supporting Information for 3 sets of FIB serial sectioning and their corresponding animations showing the serial imaging (Animations 3-5) for a specimen tested in air that highlight the 3D nature of the voids. Oxygen maps using EDS have clearly identified the voids to be oxygen rich for the tests in air (see example in Figure 5(j)), which is not observed for the tests in vacuum, implying that oxygen plays a significant role in the formation of these voids.

Our work highlights that fatigue crack nucleation and propagation are controlled by the formation of voids, either underneath surface extrusions or ahead of the crack tip, and are 
associated with ultraslow crack growth $\left(\sim 10^{-14} \mathrm{~m} /\right.$ cycle in vacuum, $10^{-12} \mathrm{~m} /$ cycle in air $)$. The formation of voids at room temperature has already been documented in the fatigue studies of bulk metals $^{21,22}$ as well as of metallic thin films on substrates, $5,6,23$ and results from the condensation of vacancies that form in the active slip bands under cyclic loading. In the particular case of metallic thin films on substrate, the voids preferentially form at the film/substrate interface, presumably because that interface does not act as a sink for vacancies. ${ }^{23}$ In persistent slip bands (PSBs), vacancies usually arise from the irreversible plastic deformation either by jog dragging on screw dislocations moving in the channels, or annihilation of edge dislocation dipoles in the walls. $^{24-27}$ Several fatigue crack initiation mechanisms have been proposed based on the presence of these excess vacancies within the PSBs, invoking either the formation of critical-radius voids ${ }^{28}$, stress concentration developing at the surface near the edges of the extrusions, ${ }^{29}$ or the formation of intrusions resulting from vacancy-diffusion-related residual stresses. ${ }^{30}$ Void-controlled fatigue crack nucleation has also been observed in ultrafine grained (UFG) $\mathrm{Cu}$ in the $\mathrm{HCF} / \mathrm{VHCF}$ regime. ${ }^{31,32}$ In that case, cyclic slip of an individual slip band led to the formation of voids along that band, especially in the VHCF regime. Our results suggest a fatigue crack nucleation mechanism similar to that invoked for VHCF of UFG $\mathrm{Cu}$, relying on void formation along individual slip bands underneath the extrusions.

In bulk macroscopic metals, fatigue cracks that nucleate at the surface first grow by single shear, leading to a zig-zag, stage I crack path, before transitioning to stage II crack growth that is characterized by Paris' law and large crack growth rates (from $1 \mathrm{~nm}$ to $1 \mu \mathrm{m} /$ cycle) ${ }^{17} \mathrm{In}$ the stage I regime, the cracks are typically microstructurally small, and their particular growth behavior (much larger rates, with respect to stage II cracks, that decrease with crack size) ${ }^{33}$ is dictated by 
the absence of closure effects and the barrier effect of grain boundaries ahead of the crack tip. ${ }^{33-35}$ Fatigue crack growth rates ranging from $10^{-10}$ to $10^{-6} \mathrm{~m} /$ cycle have also been measured for long cracks in thin metallic films under tension-tension cyclic loading. ${ }^{10,11,36,37}$ Instead, the growth of the microstructurally small cracks in our $\mathrm{Ni}$ microbeams is characterized by ultralow rates, emphasizing that the governing mechanism is unlikely to be associated with large enough cyclic plastic deformation at the crack tip (associated with irreversible emission of dislocations). In fact, the FIB cross-section SEM images suggest crack growth controlled by void formation ahead of the crack tip and linkage of these voids with the main crack. We hypothesize that this ultraslow crack growth mechanism is a direct consequence of size effects governing the fatigue behavior of these Ni microbeams. It is likely that the stress concentration effect at the tip of a growing fatigue crack is greatly reduced by the effect of the extreme stress gradients (the distance from the surface to the neutral axis is $\sim 6 \mu \mathrm{m}$ ), thereby preventing any significant cyclic crack tip opening displacement. For example, it is well known that non-propagating cracks can form in sharp notches for bulk metals. ${ }^{38}$ The underlying reason can be understood using the linear elastic or elastic plastic fracture mechanics (LEFM/EPFM) framework to show that the driving force (for example stress intensity factor range, $\Delta K$, in the case of LEFM) initially decreases with increase crack size for sharp enough notches (e.g. below $1 \mathrm{~mm}$ semicircular notches). ${ }^{39,}{ }^{40}$ It should be noted that the normalized stress gradients ahead of sharp notches in bulk metals (at most $\sim 1 \% / \mu \mathrm{m}$ ) are much smaller than in our microbeams $\left(\eta=\frac{1}{\sigma_{\max }} \frac{d \sigma}{d x}=17 \% / \mu m\right)$. Hence it is logical to assume that the driving force for fatigue crack extension in the microbeams (which cannot be quantified with the LEFM or EPFM frameworks that are not valid at these scales under these extreme stress gradients) is drastically reduced with increasing crack size. As a result, the limited amount of cyclic slip 
ahead of the crack tip may only allow vacancy formation. Hence, the ultraslow rates are likely the result of an incubation time required between crack growth events to aggregate and condensate vacancies into voids of a critical size. This explanation is consistent with the significant effect of normalized stress gradients (i.e. microbeam geometry) on the resulting $S$ - $N$ curves. ${ }^{16}$

These results also emphasized the critical effect of air on the fatigue properties, with one order of magnitude longer fatigue crack initiation life and two orders of magnitude slower crack growth rates in vacuum. For stage I crack growth associated with the common crack opening mode, the reported environmental effects are associated with mechanisms involving chemisorbed oxygen at the surface of newly exposed slip steps that increases the slip irreversibility at the crack tip, thereby preventing re-welding. ${ }^{41-43}$ Instead, in our study both fatigue crack nucleation and propagation are dominated by void formation, and the surface of the voids was shown to be oxygen rich for the tests in air. Irradiation studies (that introduce large concentrations of vacancies) of metals at large temperatures have shown that oxygen stabilizes void nucleation compared to the other vacancy cluster defects, by decreasing the void surface energy through a chemisorption process. ${ }^{44-48} \mathrm{We}$ therefore expect a similar scenario during the fatigue damage of the Ni microbeams. In air, the formation of voids is facilitated by the presence of oxygen (which is consistent with our observation of large oxygen concentrations at the location of the voids), resulting in faster crack growth rates. The void formation is likely to be limited to the surface regions and near the crack tip due to the small diffusion distance for oxygen at room temperature. In vacuum, oxygen is present at much lower concentrations (either on the surface of the specimens or as impurities in the electroplated $\mathrm{Ni}$ ), requiring longer times or larger concentration of vacancies to stabilize the voids, and resulting in much lower crack growth rates. 
The conclusions from our research has deep implications for the high / very high cycle fatigue life estimations of micro- and nano-scale devices since most modeling approaches assume a continuous crack growth mechanisms based on dislocation emission. Instead, our results suggest that modeling approaches should explore the rate of production of vacancies and voids which dictate crack growth. Future efforts will investigate multiscale modelling efforts that predict fatigue crack growth based on dislocation emission or void nucleation.

\section{ASSOCIATED CONTENT}

\section{Supporting Information.}

Supporting Information document:

Section A: In situ SEM Experimental Technique

Section B: Environmental Effects on Crack Growth Rates for a Single Specimen

Section $\mathrm{C}$ : $\underline{\text { Crack growth rates as a function of crack size }}$

Section D: FIB Serial Sectioning and Imaging for Fatigue Specimen in Air

Animations:

Animation 1: Top Surface Crack Propagation (see Figure 4(a) for corresponding $f_{0}$ evolution curve)

Animation 2: Top Surface Crack Propagation (see Figure S3(a) for corresponding $f_{0}$ evolution $\underline{\text { curve) }}$

Animation 3-5: Serial Imaging at Three Locations of a Specimen Fatigued in Air (see Figure S4 for location of the cuts) 


\section{AUTHOR INFORMATION}

\section{Corresponding Author}

*Email: olivier.pierron@me.gatech.edu

\section{Author Contributions}

The manuscript was written through contributions of AB, GMC, and ONP. All authors have given approval to the final version of the manuscript.

\section{Funding Sources}

GMC and ONP gratefully acknowledge support from NSF through award No CMMI-1562499. 

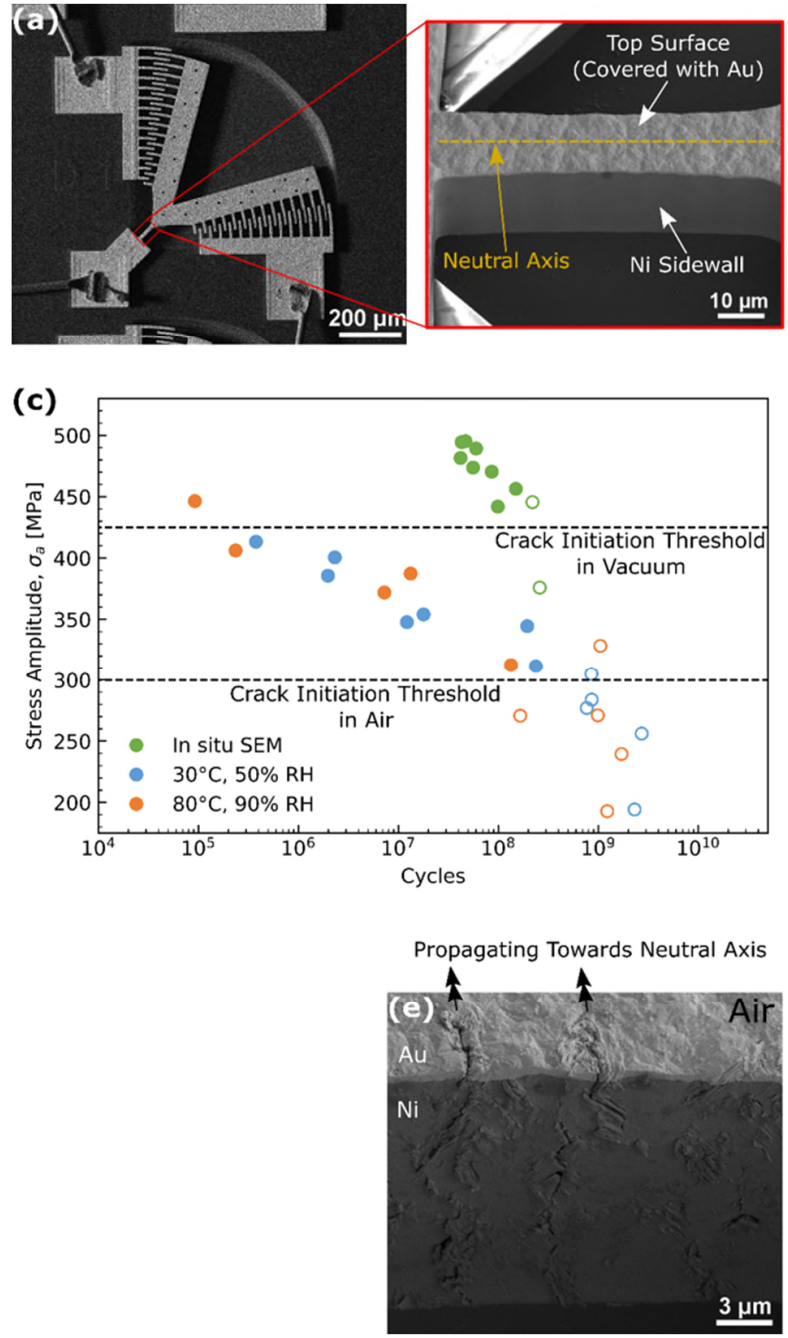
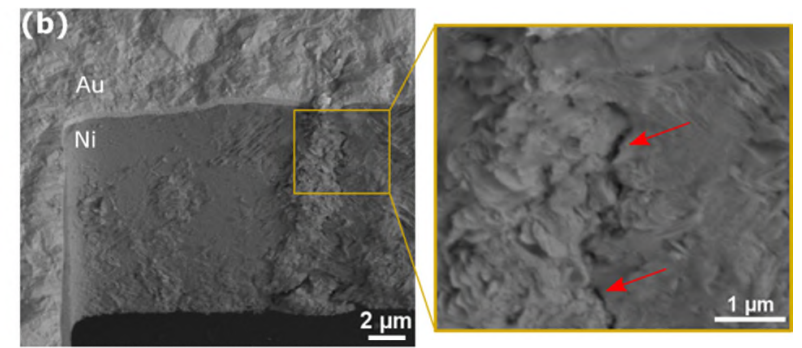

\section{(d)}

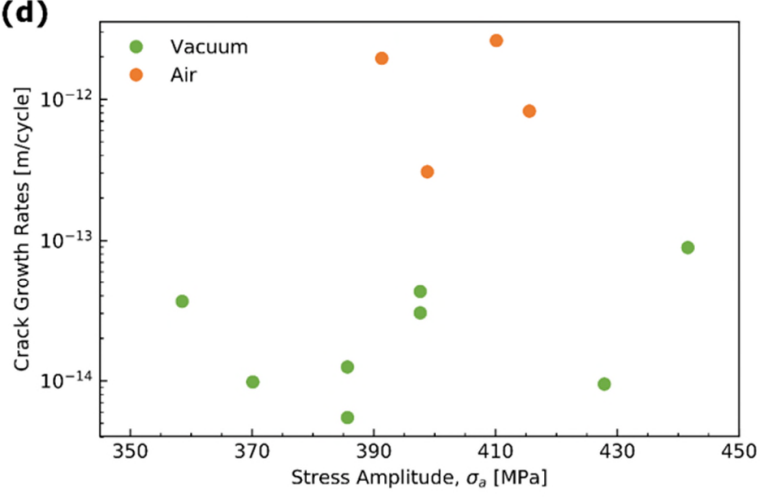

Propagating Towards Neutral Axis

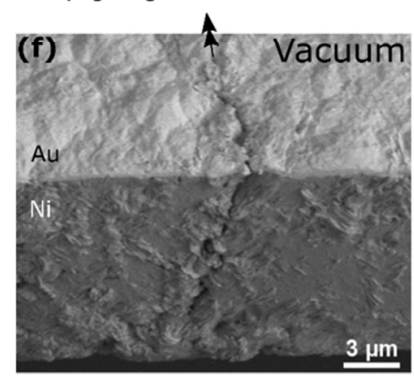

Figure 1. SEM images of (a) a Ni microresonator with an inclined high magnification image of the microbeam. (b) Inclined SEM image of a microbeam after fatigue test (in vacuum, $\sigma_{\mathrm{a}}=440 \mathrm{MPa}$, for $\mathrm{N}_{\mathrm{f}}=8.1 \times 10^{7}$ cycles) showing details of the microbeam sidewall and the extrusions formed. (c) S-N curves in air and vacuum. Empty symbols correspond to runouts specimens. (d) Crack propagation rates measured with SEM images, as a function of $\sigma_{\mathrm{a}}$ in air and vacuum. SEM images of fatigue cracks along the microbeam sidewall and propagating towards the neutral plane (see arrows) in (e) air and (f) vacuum. 
(a)

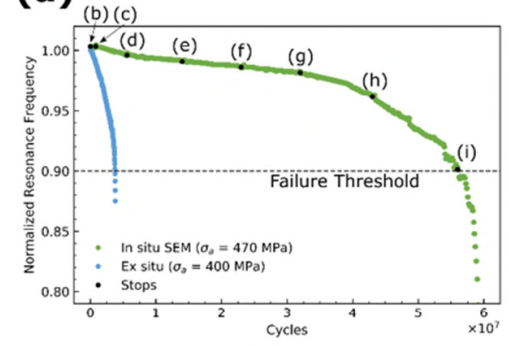

(d)

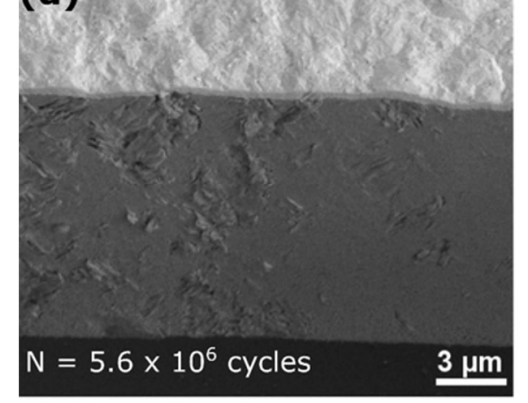

\section{(g)}

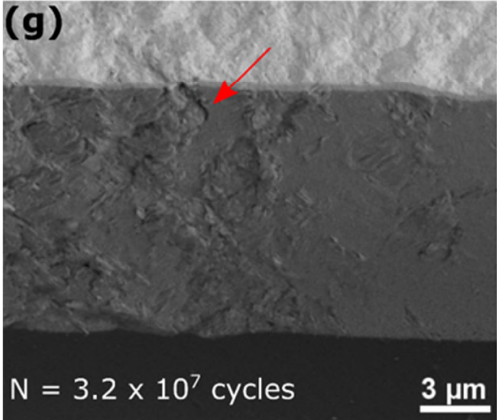

(b)
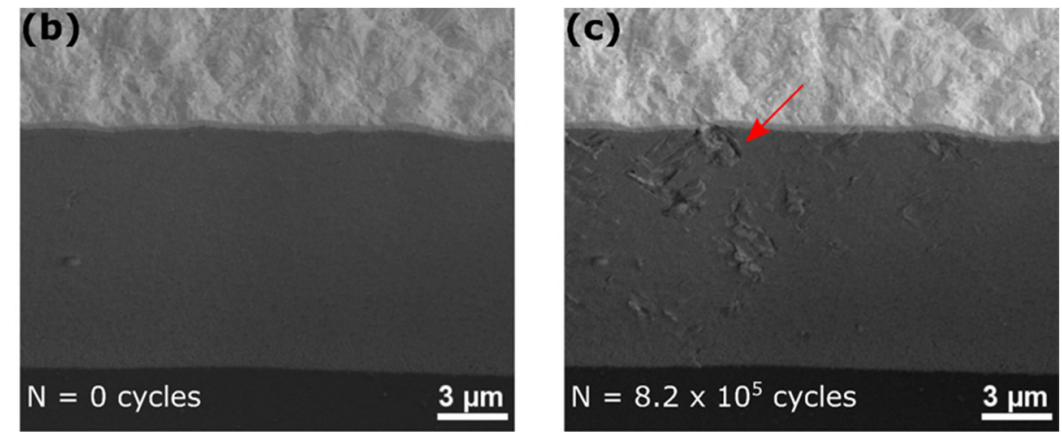

(e)

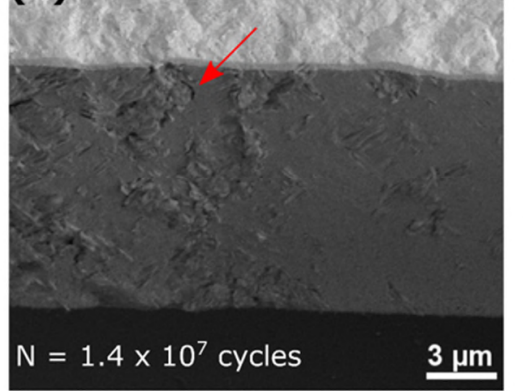

(h)

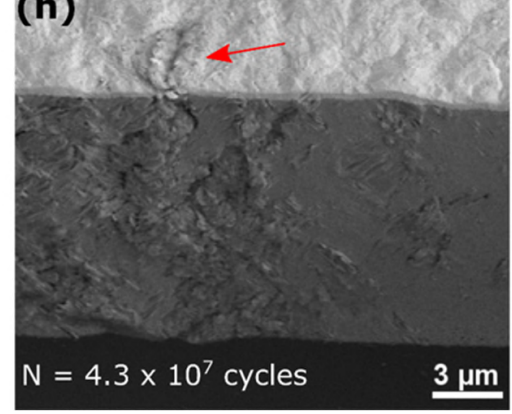

(f)

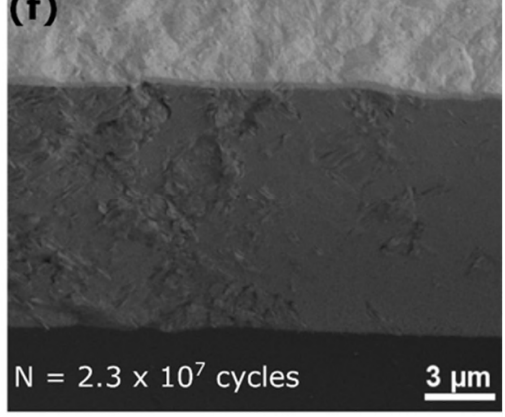

(i)

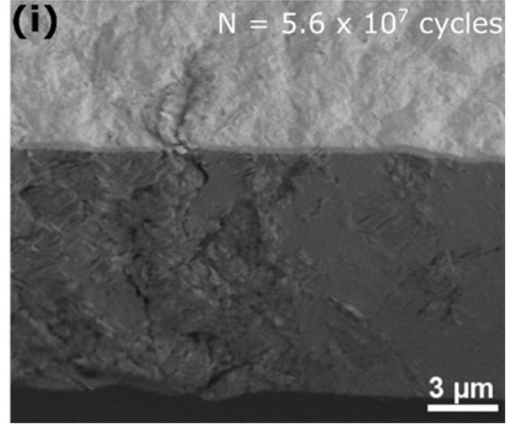

Figure 2. (a) Normalized resonance frequency evolution $\left(\frac{f_{o}}{f_{0, i}}\right)$ during an in situ SEM test performed at $\sigma_{\mathrm{a}}=470 \mathrm{MPa}$ and an ex situ test in air at $\sigma_{\mathrm{a}}=400 \mathrm{MPa}$. (b) to (i) Inclined SEM images showing the evolution of the damage along the microbeam sidewall, at increasing numbers of cycles shown in (a). 
(a)

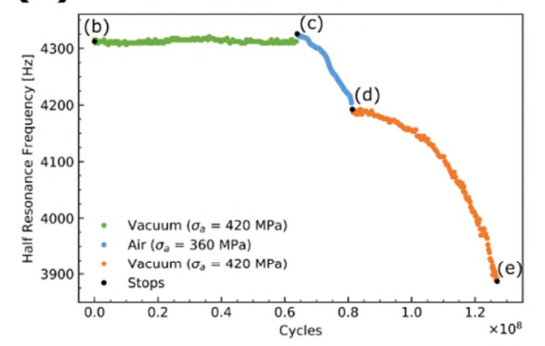

(b)

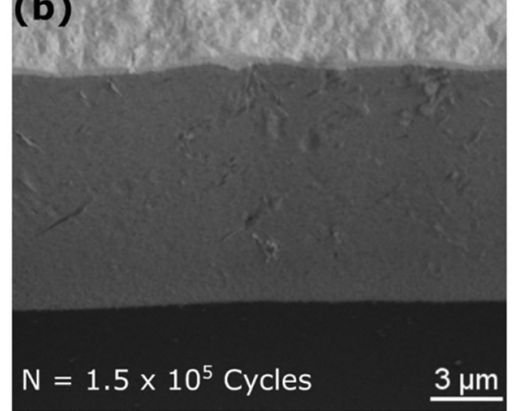

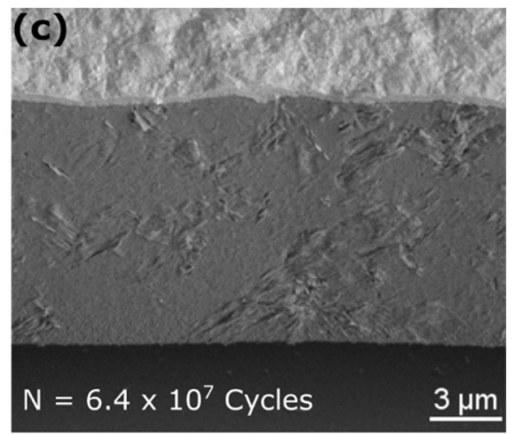
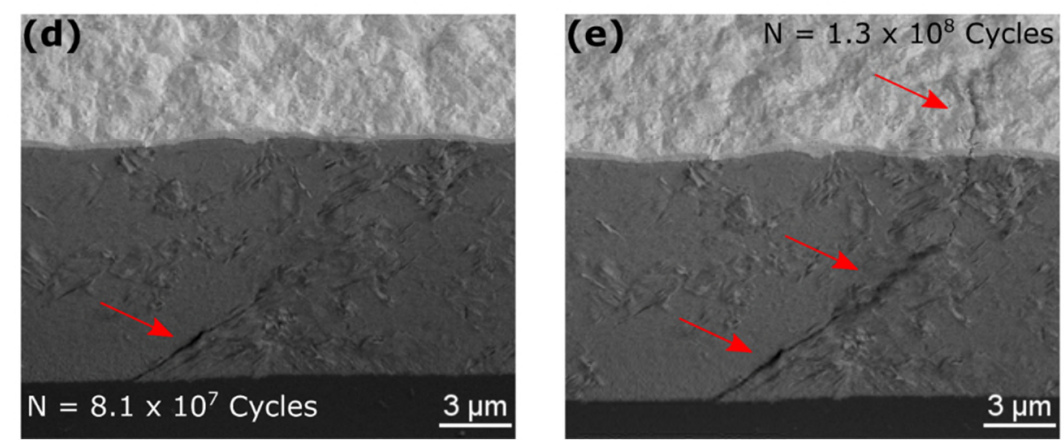

Figure 3. (a) Frequency evolution $\left(\frac{1}{2} f_{o}\right)$ during an in situ SEM test performed at $\sigma_{\mathrm{a}}=420 \mathrm{MPa}$ followed by ex situ testing in air at $\sigma_{\mathrm{a}}=360 \mathrm{MPa}$ and back to vacuum at $\sigma_{\mathrm{a}}=420 \mathrm{MPa}$. (b) to (e) Inclined SEM images showing the evolution of the damage along the microbeam sidewall. Each image was taken at the beginning and upon a change of loading conditions as shown in black in (a). 


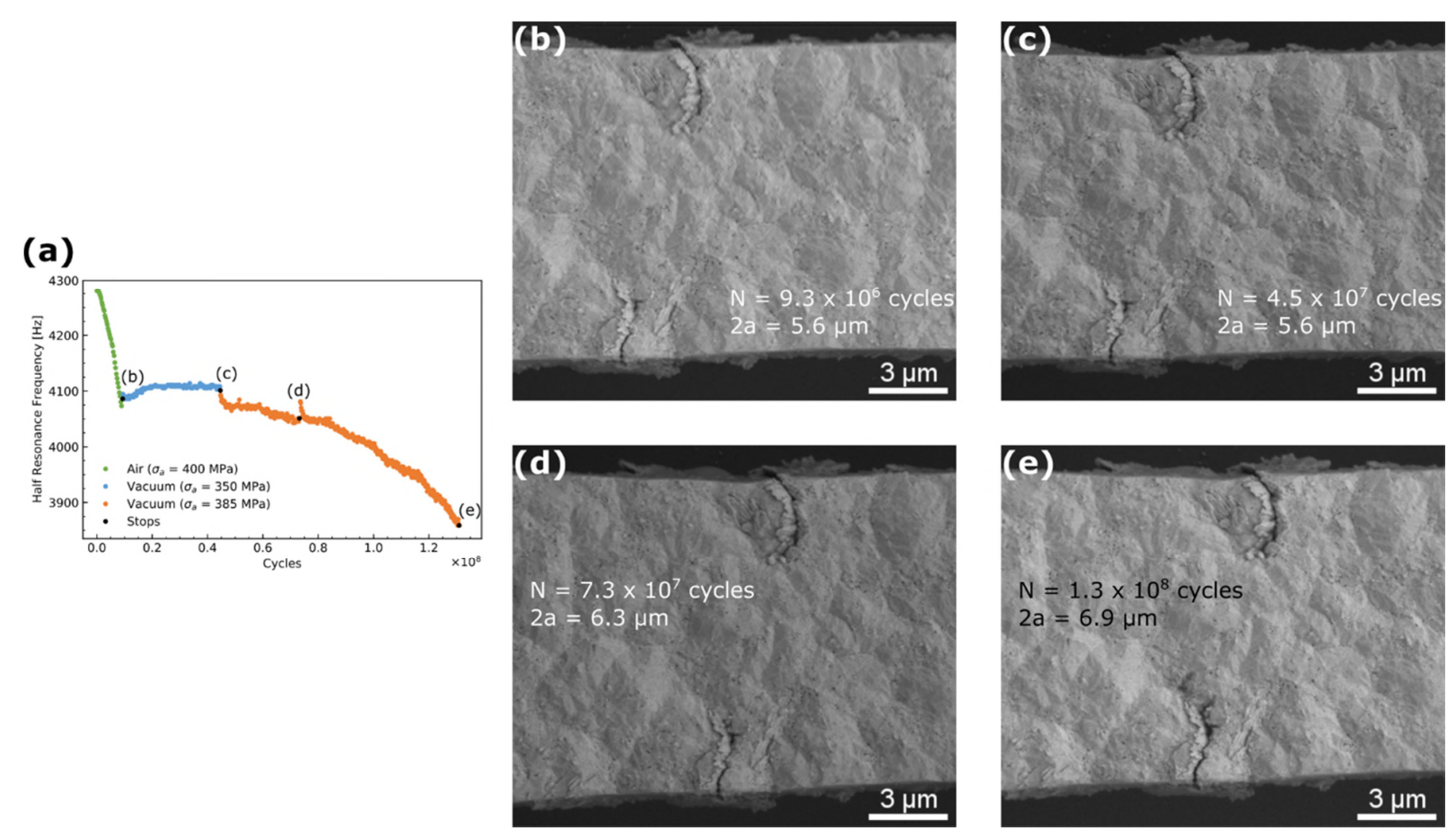

Figure 4. (a) Frequency evolution $\left(\frac{1}{2} f_{o}\right)$ during an ex situ test in air performed at $\sigma_{\mathrm{a}}=400 \mathrm{MPa}$ followed by in situ SEM testing in vacuum at $\sigma_{\mathrm{a}}=350 \mathrm{MPa}$ and $385 \mathrm{MPa}$. (b) to (e) Top-down SEM images of the microbeam's top surface, highlighting the propagation of the crack towards the neutral axis. Each image was taken upon a change of loading conditions as shown in black in (a). Note that cracks on both side of the microbeam do not increase in size between (b) and (c) (test in vacuum at $\sigma_{\mathrm{a}}=350 \mathrm{MPa}$ ). 
(a)
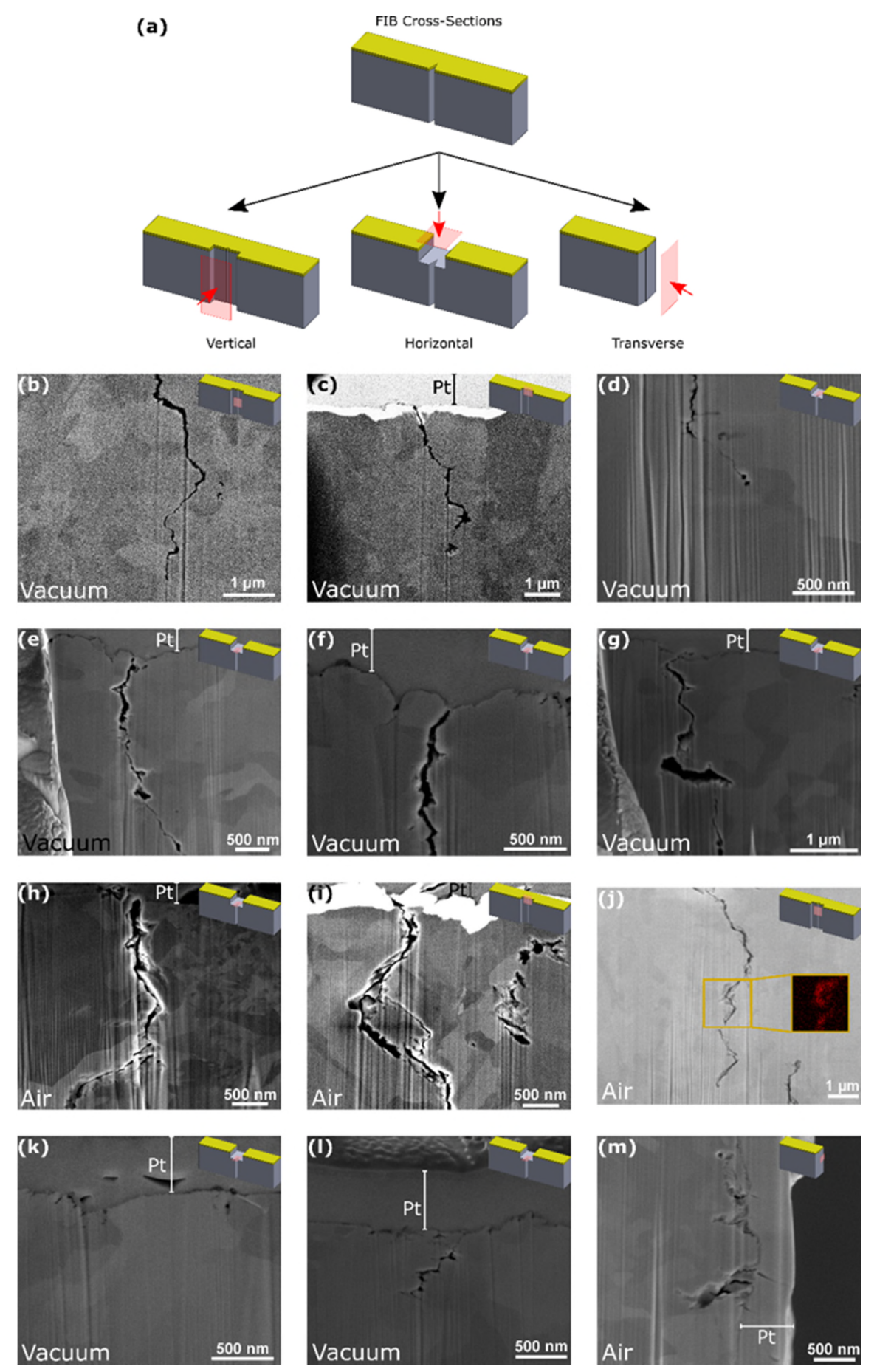

Figure 5. (a) Schematic presenting the different types of FIB cross-sections. (b) to (g) show cracks and voids for in situ SEM tests. (h) and (i) show cracks and voids for tests in air. (j) presents 
the results of oxygen concentration from EDS scans along the crack of a specimen tested in air. Voids formed in the proximity of extrusions in (k) and (l) vacuum and in (m) air.

\section{REFERENCES}

1. Newman, P. Internet of Things 2017; 2017.

2. Boyce, B. L.; Michael, J. R.; Kotula, P. G. Acta Materialia 2004, 52, (6), 1609-1619.

3. Sim, G.-D.; Krogstad, J. A.; Reddy, K. M.; Xie, K. Y.; Valentino, G. M.; Weihs, T. P.; Hemker, K. J. Science Advances 2017, 3, (6).

4. $\quad$ Baumert, E. K.; Pierron, O. N. J. Microelectromech. Sys. 2013, 22, (1), 16-25.

5. Burger, S.; Eberl, C.; Siegel, A.; Ludwig, A.; Kraft, O. Sci. Technol. Adv. Mater. 2011, $12,(5), 7$.

6. Schwaiger, R.; Kraft, O. Scripta Materialia 1999, 41, (8), 823-829.

7. Straub, T.; Berwind, M. F.; Kennerknecht, T.; Lapusta, Y.; Eberl, C. Exper. Mech. 2015, $55,(7), 1285-1299$.

8. Wimmer, A.; Heinz, W.; Detzel, T.; Robl, W.; Nellessen, M.; Kirchlechner, C.; Dehm, G. Acta Materialia 2015, 83, 460-469.

9. Wimmer, A.; Leitner, A.; Detzel, T.; Robl, W.; Heinz, W.; Pippan, R.; Dehm, G. Acta Materialia 2014, 67, 297-307.

10. Kondo, T.; Imaoka, T.; Hirakata, H.; Sakihara, M.; Minoshima, K. Acta Materialia 2013, 61, (16), 6310-6327.

11. Meirom, R. A.; Clark, T.; Polcawich, R.; Pulskamp, J.; Dubey, M.; Muhlstein, C. L. Physical Review Letters 2008, 101, (8), 4.

12. Bufford, D. C.; Stauffer, D.; Mook, W. M.; Asif, S. A. S.; Boyce, B. L.; Hattar, K. Nano Letters 2016, 16, (8), 4946-4953.

13. Lavenstein, S.; Crawford, B.; Sim, G.-D.; Shade, P. A.; Woodward, C.; El-Awady, J. A. Acta Materialia 2018, 144, (Supplement C), 154-163.

14. Eisenhut, L.; Schaefer, F.; Gruenewald, P.; Weiter, L.; Marx, M.; Motz, C. Int. J. Fat. 2017, 94, 131-139.

15. Kapp, M. W.; Kremmer, T.; Motz, C.; Yang, B.; Pippan, R. Acta Materialia 2017, 125, 351-358.

16. Sadeghi-Tohidi, F.; Pierron, O. N. Extreme Mechanics Letters 2016, 9, 97-107.

17. Suresh, S., Fatigue of materials. Cambridge University Press: 2001.

18. He, S. Y.; Chang, J. S.; Li, L. H.; Ho, H. Sens. Actuator A-Phys. 2009, 154, (1), 149-156.

19. Sadeghi-Tohidi, F.; Pierron, O. N. Appl. Phys. Let. 2015, 106, (20), 201904.

20. Sadeghi-Tohidi, F.; Pierron, O. N. Acta Materialia 2016, 106, 388-400.

21. Kettunen, P. O.; Lepisto, T.; Kostorz, G.; Goltz, G. Acta Metal. 1981, 29, (6), 969-972.

22. Grobstein, T. L.; Sivashankaran, S.; Welsch, G.; Panigrahi, N.; McGervey, J. D.; Blue, J. W. Materials Science and Engineering a-Structural Materials Properties Microstructure and Processing 1991, 138, (2), 191-203.

23. Schwaiger, R.; Dehm, G.; Kraft, O. Phil. Mag. 2003, 83, (6), 693-710.

24. Differt, K.; Essmann, U.; Mughrabi, H. Philos. Mag. A-Phys. Condens. Matter Struct. Defect Mech. Prop. 1986, 54, (2), 237-258. 
25. Essmann, U.; Gosele, U.; Mughrabi, H. Philos. Mag. A-Phys. Condens. Matter Struct. Defect Mech. Prop. 1981, 44, (2), 405-426.

26. Polak, J. Materials Science and Engineering 1987, 92, 71-80.

27. Polak, J. Materials Science And Engineering A-Structural Materials Properties Microstructure And Processing 2007, 468, 33-39.

28. Hsiung, L. M.; Stoloff, N. S. Acta Metal. Mater. 1990, 38, (6), 1191-1200.

29. Mughrabi, H. Metall. Mater. Trans. A-Phys. Metall. Mater. Sci. 2009, 40A, (6), 12571279.

30. Man, J.; Obrtlik, K.; Polak, J. Phil. Mag. 2009, 89, (16), 1295-1336.

31. Kunz, L.; Lukas, P.; Navratilova, L. Int. J. Fat. 2014, 58, 202-208.

32. Lukas, P.; Kunz, L.; Navratilova, L.; Bokuvka, O. Materials Science And Engineering AStructural Materials Properties Microstructure And Processing 2011, 528, (22-23), 7036-7040.

33. Miller, K. J. Fatigue \& Fracture of Engineering Materials \& Structures 1993, 16, (9), 931-939.

34. Castelluccio, G. M.; McDowell, D. L. International Journal of Damage Mechanics 2014, 23, (6), 791-818.

35. Castelluccio, G. M.; Musinski, W. D.; McDowell, D. L. Current Opinion in Solid State \& Materials Science 2014, 18, (4), 180-187.

36. Kondo, T.; Hirakata, H.; Minoshima, K. Int. J. Fat. 2017, 103, 444-455.

37. Meirom, R. A.; Clark, T. E.; Muhlstein, C. L. Acta Materialia 2012, 60, (3), 1408-1417.

38. Lukas, P.; Klesnil, M. Materials Science and Engineering 1978, 34, (1), 61-66.

39. Elhaddad, M. H.; Topper, T. H.; Smith, K. N. Engin. Fract. Mech. 1979, 11, (3), 573584.

40. Hammouda, M. M.; Smith, R. A.; Miller, K. J. Fatigue of Engineering Materials and Structures 1979, 2, (2), 139-154.

41. Polak, J.; Liskutin, P. Fatigue \& Fracture of Engineering Materials \& Structures 1990, 13, (2), 119-133.

42. Wood, W. A. Phil. Mag. 1958, 3, (31), 692-699.

43. Sriram, T. S.; Fine, M. E.; Chung, Y. W. Acta Metal. Mater. 1992, 40, (10), 2769-2780.

44. Zinkle, S. J.; Lee, E. H. Metallurgical Transactions a-Physical Metallurgy and Materials Science 1990, 21, (5), 1037-1051.

45. Zinkle, S. J.; Wolfer, W. G.; Kulcinski, G. L.; Seitzman, L. E. Philos. Mag. A-Phys. Condens. Matter Struct. Defect Mech. Prop. 1987, 55, (1), 127-140.

46. Seitzman, L. E.; Dodd, R. A.; Kulcinski, G. L. Metallurgical Transactions a-Physical Metallurgy and Materials Science 1990, 21, (7), 1839-1846.

47. Seitzman, L. E.; Wang, L. M.; Kulcinski, G. L.; Dodd, R. A. Journal of Nuclear Materials 1986, 141, 738-742.

48. Cawthorne, C.; Fulton, E. J. Nature 1967, 216, (5115), 576-+. 
Abstract Figure

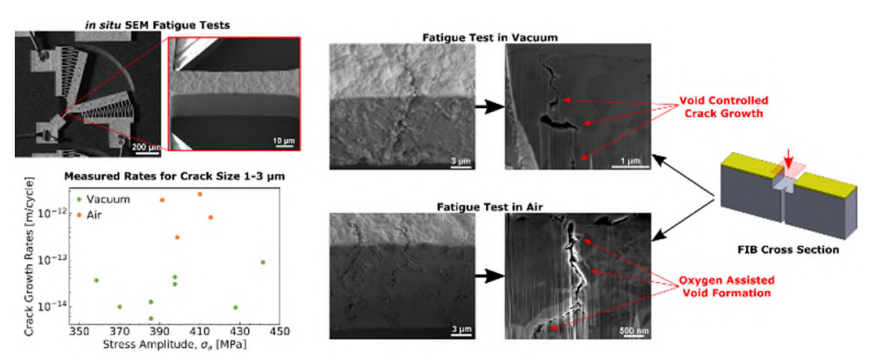




\section{Quantitative in situ SEM high cycle}

fatigue: The critical role of oxygen on

nanoscale-void-controlled nucleation

and propagation of small cracks in Ni microbeams

\section{Barrios, Alejandro}

Alejandro Barrios, Saurabh Gupta, Gustavo M. Castelluccio, and Olivier N. Pierron. Quantitative in situ SEM high cycle fatigue: The critical role of oxygen on nanoscale-void-controlled nucleation and propagation of small cracks in Ni microbeams. Nano Letters, 2018, Volume 18, pÿlssue 4, pp. 25952602

http://dx.doi.org/10.1021/acs.nanolett.8b00343

Downloaded from Cranfield Library Services E-Repository 